\title{
Rural women's knowledge of prevention and care related to breast cancer
}

\author{
$\mathrm{NH}$ Mugivhi \\ Tshwane University of Technology \\ JE Maree, RN D Cur (Pret) \\ Associate Professor and Head: Adelaide Tambo School of Nursing Science, Tshwane University of Technology \\ SCD Wright, RPN, D Tech Nursing \\ Associate Professor : Adelaide Tambo School of Nursing Science, Tshwane University of Technology
}

\section{Kev words}

Breast cancer, signs and symptoms of breast cancer, breast self-examination, health care provider, rural women

\section{Correspondence address}

Lize Maree

Tshwane University of Technology

Private Bag X680

Pretoria 0001

Tel: (012) 382-5285

Fax:(012) 382-5033

Mobile: 0833625749

E-mail :mareeje@tut.ac.za

\section{Abstract: Curationis 32 (2): $38-45$}

According to the experience of the researcher, an oncology nurse, women living in the rural areas of Thulamela municipality in the Limpopo Province, have many different perceptions of breast cancer. Perceptions are based on previous disease experiences. As with previous illnesses, changes in the breast caused by breast cancer are self-managed and treated. When these women seek medical advice for breast cancer related problems, they already have advanced cancer. The purpose of the study was to investigate if women are knowledgeable of the signs and symptoms of breast cancer, breast self-examination, as well as appropriate health care to take responsibility to prevent admission with advanced breast cancer. The research study was an exploratory and contextual survey. The sampling method was convenient $(n=200)$. Data were gathered during a structured interview using a checklist. Data analysis was done by means of descriptive statistics. The results of the study indicated a low level of knowledge regarding the signs and symptoms of breast cancer. The average level of knowledge for the signs and symptoms of breast cancer was less than $10 \%$ $(n=20)$. With regards to breast self-examination the results varied between $8.5 \%$ $(n=17)$ and $13 \%(n=26)$. Biomedical medicine was the preferred treatment choice for the majority of the respondents. The study provided evidence that women were unable to take responsibility for their breast health. Their lack of knowledge of the signs and symptoms of breast cancer and breast self-examination would not enable them to prevent presenting with advanced disease. A breast health care strategy for women living in Thulamela should be designed, implemented and evaluated to prevent presentation with advanced breast cancer.

\section{Background, rationale and problem statement}

Breast cancer is a malignancy which is easily treated and cured if detected early. However, if detected late, the prognosis is unfavourable (CraneOkado, 2001:129). Previously, women lacked strategies for detecting breast cancer early, but at present, through breast self-examination, a clinical breast examination, or mammogram, changes in the breast can be detected at a relatively early stage.

Though these strategies are widely publicised, the high incidence of breast cancer is still a concern. According to the World Health Organization and International Union Against Cancer (2005), breast cancer is the most common cancer among women worldwide. One in eight American women will develop breast cancer (Smyke, 1993:95). In Africa, the problem is equally con- 
cerning. The Cape Town Declaration on Cancer Control in Africa (2006), urged governments in the African region to recognise cancer as a critical public health problem alongside HIV/ AIDS, malaria and tuberculosis.

In South Africa, The Cancer Association of South Africa reports that breast cancer is one of the most common cancers affecting women (CANSA, 2004:1). The Medical Research Council (2006) reported that 3156 women died in 2000 due to breast cancer, and that breast cancer was the second-ranked cause of cancer deaths in females in South Africa. Though black women in South Africa have a low incidence of breast cancer, the incidence is rising (Walker, Adam \& Walker, 2004:81). The authors also report that in those women who were admitted, the mean age was relatively young ( 54.1 years of age, with a margin of error of 10.9 years) and they typically presented with advanced progression of the disease. Vorobiof, Sitas and Vorobiof (2001:125s) state that several determinants influence rural women in their decision to obtain early medical help, as well as to refrain from proposed therapeutic methods.

One determinant is a negative attitude towards the disease. After detecting an abnormality, some women block their anxiety from their conscious awareness and claim that they did not notice that something was wrong in their bodies. This behaviour causes a delay in the treatment of patients who could benefit from early treatment. Some women also refuse to give consent to treatment, while others wait and think about the situation or deliberately delay taking any action (Barraclough, 1996:17).

Lack of knowledge has been identified as a determinant that creates problems with the management of the patients (Barraclough, 1996:6), and is particularly evident with regards to the additional signs and symptoms of breast cancer. While Barraclough (1996:6) observes that some women knew a lump in the breast can be a sign of breast cancer, they do not know that other symptoms, such as a nipple discharge or inversion, may also indicate breast cancer. Vorobiof and Vorobiof (2001:126s) found the idea of a painless lump in the breast being potentially cancerous and therefore lifethreatening a difficult concept for many black women to accept.

According to Donegan and Spratt (1995:8), women who lack knowledge about breast cancer may be surprised if someone who appears to be well is diagnosed with breast cancer. The diagnosis of breast cancer may also be traumatic if it is made during screening and the person was not aware of any symptom of the disease. Some women accept the diagnosis and treatment with a fighting spirit, while others are affected by both the emotional and cultural meaning regarding the breasts.

Unfortunately Black women with breast cancer present with far advanced disease (Walker 2004). Walker (2004) found that $21.1 \%$ of women presented with stage III and $63.1 \%$ presented with stage IV breast cancer. Early detection and treatment are the only weapons women have to prevent death due to breast cancer. The successful outcome of breast cancer depends heavily on early diagnosis and treatment. Self-examination of the breasts is an important tool for women to detect changes in their breasts as a first line of defence against breast cancer. Equally important is the decision where to obtain health care if a problem is suspected. If a woman is not knowledgeable about the signs of breast cancer, she will interpret the signs from her own previous experience which could delay obtaining appropriate health care. .

\section{Problem statement}

The researcher, an oncology nurse, experienced that women living in the rural areas of Thulamela municipality in the Limpopo Province, have many different perceptions of breast cancer. These perceptions are based on their experience of previous diseases. As with previous diseases they typically resort to self-management and treatment. Some women even associate breast cancer with a chronic ulcer (Tshipfula) or an abscess which they can treat themselves.

When they do eventually seek medical advice, the cancer has progressed to an advanced stage, putting them beyond medical treatment to cure the disease. Cultural perceptions also exist that may affect a delay in seeking medical advice, as removal of one or both breasts is often unacceptable if a woman is to remain attractive to her partner.

The research problem for the study was therefore to investigate if women of the Thulamela municipality in the Limpopo Province are knowledgeable of the signs and symptoms of breast cancer, breast self-examination, and appropriate health care, so as to prevent admission with advanced breast cancer.

\section{Purpose of the study}

The purpose of the study was to determine the knowledge of women in the Thulamela municipality regarding the signs and symptoms of breast cancer, breast self-examination, as well as their decision of where they will obtain health care. This knowledge will be a prerequisite to develop culture-specific health promotion interventions for educating rural women living in the Thulamela municipality in the Limpopo Province regarding breast cancer.

\section{Theoretical framework}

Orem's self-care theory was used to guide this study (Berbiglia, 2006:255). Orem's theory was used because the theorist provided it as a key for enabling a person to care for him/herself. According to Leddy and Pepper (1998:179-180), the self-care theory indicates the purpose of nursing as help given to people to meet their self-care needs. Self-care is the practice of activities that individuals initiate and perform on their own behalf in maintaining life, health, and wellbeing.

There are three systems within Orem's professional nursing practice model, namely:

- the compensatory system where the nurse is responsible for total care;

- the partially compensatory system where the nurse and patient share responsibility for care; and

- the supportive-educative system where the client has primary responsibility for health with the nurse acting as a consultant.

The current study focussed primarily on the supportive-educative system. Before women can be educated, their current level of knowledge must be investigated to serve as the basis for an educational intervention. 


\section{Research methods and design}

The research methods and design will be described in terms of the context; population; design and data gathering; and data analysis.

\section{Context}

The study was contextual (LoBiondoWood \& Haber, 2006:561). Contextual means that the study results are only valid for the situation in which the study was done. The context for the study was Region 4 of the Thulamela municipality in the Limpopo Province. The area was chosen because most of the patients who were admitted with advanced breast cancer at the local hospital, Tshilidzini, lived in the area. The inhabitants of Thulamela municipality are predominantly Venda and Tsonga.

According to the Census 2001 (2001), the two main languages spoken are Tshivenda and Xitsonga. Approximately 384900 persons use Tshivenda compared to 192600 who speak Xitsonga. The population density for the area is 180 persons per square kilometre. The total black population for the area is approximately 582000 , of which $46 \%$ is male and $54 \%$ female. In terms of their educational level, $30 \%$ of the population had no schooling at all, $43.4 \%$ had some secondary education, and only $8.1 \%$ of the population had a tertiary education. The majority of the women are not employed, and the women primarily earn their living by means of self-help projects, small businesses and care group activities.

\section{Population}

The target population included accessible, healthy, rural black women of different age groups from 18 years upwards living in the Thulamela municipality. The inclusion criteria were female, Black, older than 18 years, no prior diagnosis of breast cancer, residing in Thulamela municipality and willing to participate. Convenience sampling, a form of non-probability sampling, was used. Convenience sampling entails the use of the most conveniently available women as study participants. All the women approached were part of a group activity, which could have been a care group, small business group or self-help group. Following the guideline for the sample size from the statistician at Tshwane University of Technology, 200 women were included in the study $(\mathrm{n}=200)$.

\section{Design and data gathering}

The design of the study was a quantitative survey (Burns \& Grove, $2001: 256)$. The data gathering method for the study was a self-report method using a structured interview. According to Burns and Grove (2005:396) a structured interview has the benefit of allowing the interviewer to explain or rephrase the questions so that the participants can better understand the question. As a result of the high rate of illiteracy among the women, a selfcompleted questionnaire would not have been feasible.

The data gathering instrument was a checklist. Polit and Hungler (1997:275) describe a checklist as an instrument used by an observer to record observed phenomena. A checklist was appropriate as the answers of the respondents provided during the structured interview were indicated on the checklist. The checklist was based on theoretical knowledge of breast self-examination and the signs and symptoms of breast cancer provided by Hamolsky and Facione (1999:432) and Crane-Okado (2001:124). The checklist consisted of two sections. Section A was devoted to the respondents' socio-economic data, and section B contained questions regarding breast self-examination, the signs and symptoms of breast cancer, as well as the choice of preferred treatment.

The checklist was pre-tested before actual data gathering took place to ensure that the data required to answer the research question were gathered. Pre-testing of a measuring instrument involves trying it out on a small number of persons with similar characteristics to those of the respondents' target group to refine the methodology including the instrument and data gathering process (Burns \& Grove, 2005:746). The checklist was pre-tested on 10 women who did not form part of the main sample. After the pre-test, five questions were added to the checklist. For the treatment of choice, an option was added in terms of faith healers, as well as an "other" choice to be able to add options not listed.

\section{Data analysis}

The results of the checklist were analysed through descriptive statistics and presented by means of frequency tables and figures. Burns and Grove (2001:794) define descriptive statistics as the organisation of data in ways that give meaning and facilitate insight, such as frequency distributions and measures of central tendency and dispersion. Data were analysed using the SPSS 13.0 programme.

\section{Ethical considerations}

According to Brink (2002:38), it is every researcher's responsibility to ensure that the ethical code is maintained at all times. The principle of beneficence was maintained as the researcher strived to avoid inflicting psychological harm by practicing culture-sensitive nursing. Health education regarding breast cancer was given to the total group of women after the data were collected. The principle of respect of human dignity was also preserved. The right of self-determination of the participants was acknowledged. The participants volunteered to take part in the study after being informed about it in their own language and they could also terminate their participation at any time. The interview was conducted in the preferred language of the participants. Privacy was ensured by taking the participants aside when others were present. Anonymity and confidentiality was also ensured, as no names were written on the checklists, only a sequential number. The right of the participants to fair treatment was upheld by fair and non-discriminatory selection based on research requirements. The Ethics Committee of Tshwane University of Technology approved the proposal of the study.

\section{Validity and reliability}

Validity refers to the degree to which an instrument measures what it is supposed to measure (Polit \& Hungler, 1997:471). Reliability has been defined as the accuracy and consistency of the measuring instrument. A research instrument can be considered reliable if it yields results on separate occasions under comparable conditions.

Measures that were taken to assure the validity and reliability of the study are 


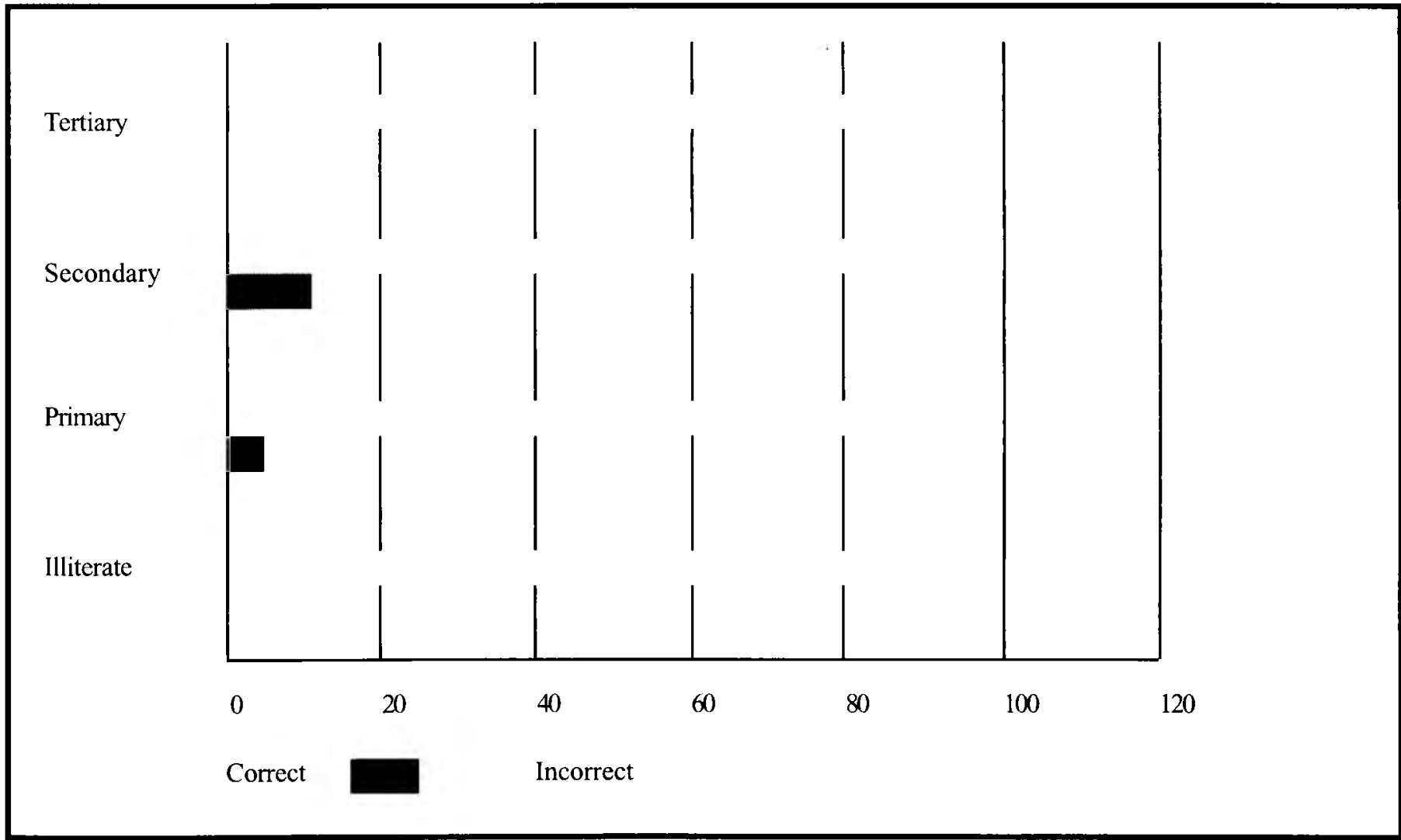

the following:

- The checklist was given to a professional nurse trained in oncology nursing to determine content validity.

- $\quad$ The checklist was pre-tested to ensure that all questions were understood and that the required data were produced.

- $\quad$ The checklist was formulated and specifically planned to address the rural black women's knowledge regarding self-examination for breast cancer, signs and symptoms of breast cancer and preferred choice of treatment.

- The researcher collected all the data, thus avoiding data gathering variability.

- The researcher was available in person to answer and explain any unclear aspects to the participants.

- The respondents were addressed in their own language to improve understanding of the questions and build trust.

- The checklist was the correct instrument to tick off the responses of the respondents, thus ensuring that all data were captured correctly.

- Using the checklist ensured structural coherence through all 200 interviews.

\section{Results}

The results of the study will be presented in the format of the checklist. The demographic data will be presented first, and thereafter the results regarding the breast self-examination, signs and symptoms of breast cancer and the choice of treatment.

\section{Demographic data}

The majority of the women $(47 \% ; n=94)$ came from small business groups, and $41.5 \%(n=83)$ from self-help groups. The distribution of the socio-cultural groups was as follows: Tsonga $26 \%$ $(n=54)$, Venda $70 \%(n=140)$ and others $4 \%(n=8)$. In terms of education, $62.7 \%$ $(n=125)$ of the women had at least some secondary schooling. In the study sample, only $7 \%(n=14)$ were completely illiterate. A small percentage, $4 \%(n=8)$, had any tertiary education. Most respondents $(38 \% ; n=76)$ were between the ages 30-39 and the majority of the respondents $(87 \% ; n=174)$ were between $20-49$ years old.

\section{Knowledge of breast self- examination}

Three specific questions were asked regarding breast self-examination. The questions focussed on the frequency of breast self-examination, the optimal time of self-examination and the palpation technique.

\section{Frequency of breast self-examina- tion}

The first question focussed on the frequency of breast self-examination. The majority of the group $(92 \% ; n=184) \mathrm{did}$ not know the correct frequency of breast self-examination. When investigated in terms of the groups from which the women were recruited, only one group, the self-help group, had $18 \%$ $(n=36)$ correct. Both the small business and self care groups gave no correct answer.

In terms of the socio-cultural groups, only a small percentage of the Venda women ( $11.5 \% ; n=16)$ knew the correct frequency of breast self-examination. Both the Tsonga and Other group had no correct answer.

When the correct frequency of breast self-examination was cross tabulated with the level of education, correct an- 
swers came only from the group who had at least some primary and secondary education. None of the women with a tertiary education knew the correct frequency of breast self-examination. The level of education and knowledge of the correct frequency of breast self-examination is illustrated in Figure 1.

\section{Time of breast self- examination}

The second question regarding the correct time of the examination revealed that of the 200 women questioned, only $8.5 \%(n=17)$ knew the correct time of examination. As in the first question, these women came from the self-help group. With regard to the socio-cultural groups, only $12 \%(n=17)$ of the Venda women were knowledgeable and none in the Tsonga or other group. In terms of education, only some women with a primary $(9.1 \% ; n=3)$ and secondary level $(9.7 \% ; n=14)$ of education answered correctly. In the age groups, only $14.4 \%(n=11)$ of the $30-39$ age group and $8 \%(n=4)$ of the $40-49$ age group were correct in their responses.

\section{Method of examination demonstrated}

The third question regarding breast self-examination focussed on the correct method of breast self-examination. Four specific aspects were investigated. These aspects were the correct palpation technique, examining the whole breast, as well as both the nipple and axilla. The results are presented in Figure 2. Again, the level of knowledge was low, the highest correct percentage being examining the whole breast $(21 \% ; n=42)$.

When the demographic variables were taken into account, the most knowledgeable women were found to be Venda women between the ages of 30 39 forming part of the self-help group and having a secondary level of education.

\section{Knowledge of the signs and symptoms of breast cancer}

Eight signs and symptoms of breast cancer were investigated. The signs and symptoms of breast cancer included in the checklist were:

- $\quad$ change in the size of the breast;

- change in the contour of the breast;

- mass or thickening;

- $\quad$ skin retraction, dimpling;

- nipple retraction;

- $\quad$ persistent rash in nipple area;

- nipple discharge; and

- $\quad$ superficial veins on the chest.

In Figure 3, an overview of the women's level of knowledge regarding the signs and symptoms of breast cancer is provided. From Figure 3, it is clear that the overall level of knowledge was low. The highest percentage is $15.5 \%$ $(n=31)$ for knowing that the size of the breast would change. Changes in the contour of the breast $(13 \% ; n=26)$ and knowledge about superficial veins on the chest $(12 \% ; n=24)$ were second and third. The least known signs were skin retraction and nipple retraction, both being $8.5 \%(n=17)$.

Regarding a mass or thickening, three possibilities were listed, namely a mass in the breast, the axilla or supraclavicular. Again, there was little variance in the level of knowledge $-16 \%(n=32)$ of the women knew of a mass in the breast and $15.5 \%(n=31)$ about a mass in the axilla. Concerning the mass or thickening in the supraclavicular area, only $7.5 \%(n=15)$ were knowledgeable.

Investigating the results in terms of the demographic variables, a definite trend was noticeable:

- In terms of the groups the women belong to, the self-help group was by far the most knowledgeable, ranging from $15.7 \%(n=13)$ to $34.9 \%(n=29)$. The level of knowledge of the small business activity and the care group is similar and quite low (below 5\%). In the sociocultural groups, the Venda was the only group with some knowledge, ranging from $10.7 \%$ $(\mathrm{n}=15)$ to $22.9 \%(\mathrm{n}=32)$.

- The educational level indicated again that having a secondary education was most profitable in terms of knowledge of breast cancer. It must be noted that the percentage for the tertiary education group might be misleading, as there were only 8 $(n=8)$ respondents in the group (12.5\% of the respondents rep- 


\section{cancer.}

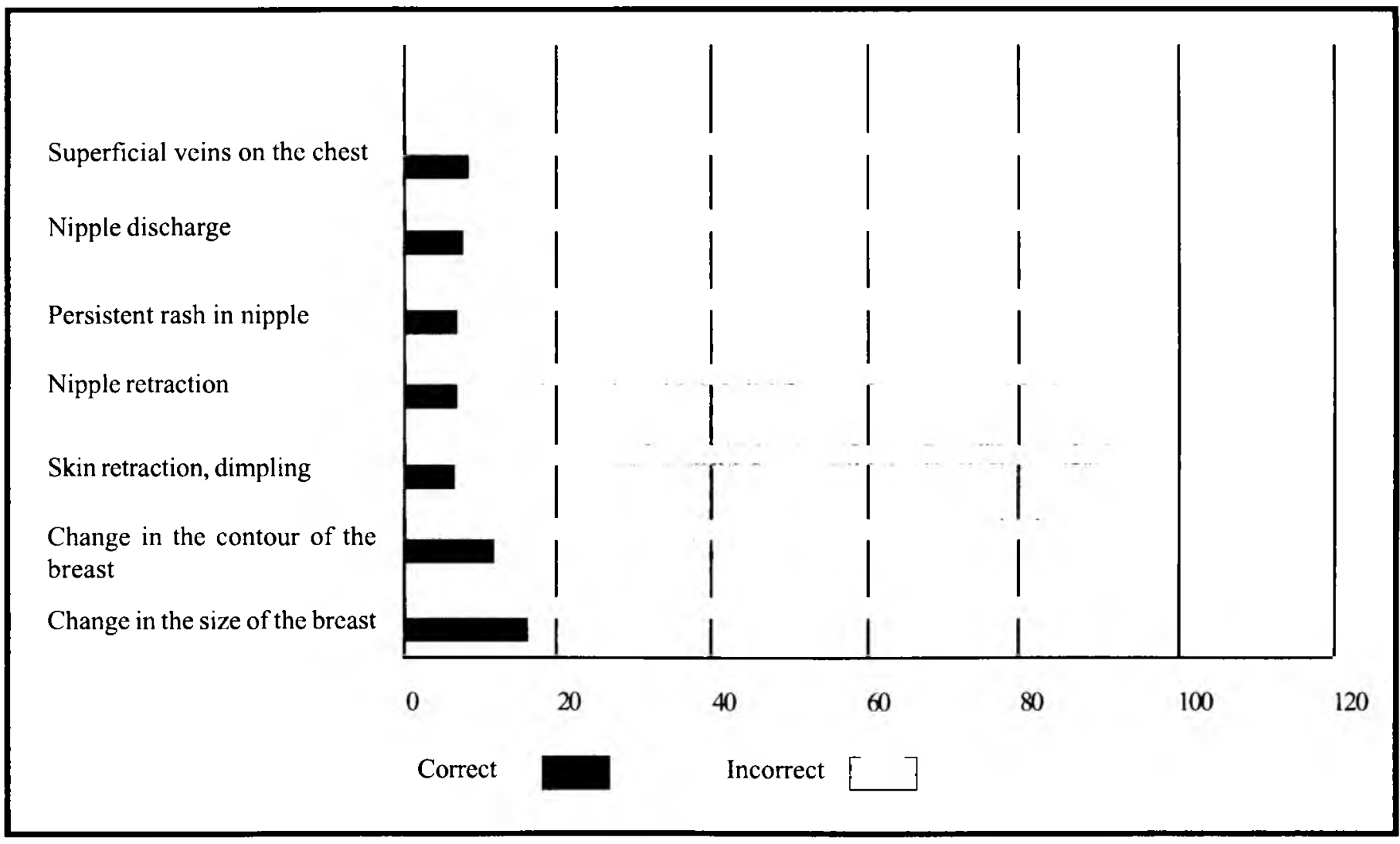

resents one respondent with a correct answer).

- $\quad$ The age groups also present a clear pattern. Those younger than 20 years of age and older than 60 years of age did not have any knowledge of breast cancer. The 30-39 age group had the most knowledgeable respondents, with a range of $11.7 \%(n=20)$ to $27.3 \%(n=48)$.

\section{Preferred treatment}

Four options were offered in the checklist. These were self treatment, traditional medicine, faith healing and biomedical treatment. An "Other" option was also provided. The results indicate that biomedical treatment was preferred by the majority $(68.5 \% ; n=139)$ of the respondents. Traditional medicine is preferred by $8 \%(n=16)$ of the group and faith healing by $5 \%(n=10)$. Only $1 \%(n=2)$ of the respondents indicated that they prefer to treat themselves.

Most of the women who chose traditional or faith healing belonged to the care group, in both cases $13 \%(n=3)$. Interestingly, only $42.6 \%(n=40)$ of the small business activity group preferred being treated biomedically. Concerning the socio-cultural group, $19.2 \%$ $(n=10)$ of the Tsonga group indicated their preference for traditional healers. An open-ended question invited the respondents to motivate their preferred choice of treatment. Almost half the group ( $43.5 \% ; n=87$ ) chose a medical practitioner as preferred treatment. Comments were that doctors know how to treat cancer; doctors specialise in treating cancer; and doctors were specifically trained to treat cancer. The hospital was mentioned by $16.5 \%$ $(n=33)$ of the respondents. The most frequent reason was that specific equipment at the hospital might be needed for the treatment, for example an X-ray machine. Another reason was also that an operation could be done if needed. Registered professional nurses were chosen by $3 \%(n=6)$ of the group. Traditional medicine $(5 \% ; \mathrm{n}=10)$, religious healing $(3.5 \% ; n=7)$, and herbal medicine $(1.5 \% ; n=3)$ were chosen infrequently. In Figure 4 the motivation is presented.

A group of women were identified by means of the open-ended question. They were not identified through the other questions on the checklist. These women $(27 \%, n=54)$ (Figure 4) motivated their preferred treatment choice with the statement that they did not know what breast cancer was. Due to the importance of the group, a full description of the group is provided.

In terms of the activity group, $77.8 \%$ $(n=42)$ were in the small business activity group. The majority $(51.9 \%$; $\mathrm{n}=28$ ) were Tsonga women, with $40.7 \%$ $(n=26)$ being Venda women. Their level of education reflected the main group's level of education with $11.1 \%(n=6)$ being illiterate, $38.9 \%(n=21)$ with a primary level of education, $48.1 \%(n=26)$ with a secondary level and $1.9 \%(n=1)$ with a tertiary level of education. The age groups were also similar to those of the main group.

The majority $(77.8 \% ; n=42)$ was between 20-49 years of age. When compared to the main group, two significant differences were found:

- The only difference between this sub group of women and the main group is that the majority were in the Tsonga and Other groups, with only $40.7 \%$ $(n=22)$ Venda. In the main group, $70 \%(n=140)$ were Venda women. 
Another important difference is that $88.9 \%(n=48)$ of the group chose a non-biomedical treatment choice.

\section{Discussion}

The study provided evidence that women living in Thulamela in the Limpopo Province had a low level of knowledge of signs and symptoms of breast cancer and breast self-examination. Less than $20 \%(n=40)$ of the group had knowledge of the signs and symptoms of breast cancer and $27 \%(n=54)$ of the group did not know about breast cancer at all. This is not exceptional as in a study conducted in 2006 to explore rural Turkish women's knowledge of breast self-examination and mammography, Dunar, Özmen, Öztürk, Haspolat, Akyildis, Çoban and Cakiroglu (2006) found that $23.4 \%$ of their sample of 224 had no knowledge of breast cancer whilst $29.7 \%$ had no concept of breast self-examination. Oluwatosin (2006:461) also reports a low level of understanding of breast cancer and self-examination among women Ibadan, Nigeria. It is interesting that women from Thulamela as well as the sample of $\mathrm{Ni}$ gerian women most commonly acknowledged a change in the size of the breast, also present with swelling, as a sign of breast cancer. A change in the size of the breast is not a sign of early breast cancer and according to Nahcivan and Secginli (2007) a lack of knowledge of breast cancer leads to a great risk for advanced breast cancer and metastatic spread.

A relatively high percentage of the women $(66.5 \% ; n=133)$ had a secondary or tertiary education. The level of education, however, was not a predictor of knowledge in terms of breast selfexamination (frequency of breast selfexamination, time of breast self-examination, method of examination) and the signs and symptoms of breast cancer. The group with a tertiary education had overall less knowledge than the group with a secondary education. This finding is supported by Dunar et al. (2006), who found that $9 \%$ of the women in their study having primary education had sufficient knowledge of breast cancer.

The age group with the best knowledge (approximately 20\%) was the 30-39 year age group. The results indicate that an inverse relationship exists between age and level of knowledge. The $60+$ age group had no knowledge at all regarding breast cancer, while at this age the prevalence would be higher than in the younger age groups. Dunar et al. (2006) did not included women of 65 and older in their study due to a perceived risk of inadequate understanding. In a study aimed at teaching women 40 years and older about breast cancer and breast self-examination, Budakoglu, Maral, Ozdemir and Bumin (2007:108) found that only $4.3 \%$ to $18.7 \%$ of the participant women $(n=462)$ had knowledge of most of the steps of breast self-examination. In terms of the groups, the selfhelp group had a considerably higher level of knowledge than the other two groups. The reason for this phenomenon should be investigated, as the group might present a viable avenue for teaching the community.

The study also provides evidence that the traditional healer is not the preferred health care choice of women living in Thulamela. This is in contrast with the findings of a study conducted in 2002 by Puckree, Mkhize, Mgobhozi and Lin (2002:247) who described that $70 \%$ of their sample of patients $(n=300)$ selected in Durban, Kwa- Zulu Natal, preferred a traditional healer.

The results indicated that the Tsonga women are less knowledgeable about breast self-examination and signs and symptoms of breast cancer. The Tsonga women were also more prone to choose non-biomedical treatment options. The reason for the difference in the two socio-cultural groups was not clear from the research. The reason might be historical and should be investigated in depth before any health promotion intervention is developed.

The Tsonga women could possibly be a vulnerable and socially disadvantaged group and like other vulnerable and disadvantage groups have fewer health resources, get sicker and die earlier than those in more privileged social positions (Irwin, Valentine, Brown, Loewenson, Solar, Brown, Koller, \& Vega, 2006). According to the afore-mentioned authors, the majority of health problems of these groups can be attributed to the social conditions in which people live and work. These social determinants will have to be investigated and incorporated in a health promotion intervention specifically developed for Tsonga women.

\section{Limitations}

The limitations of the study were the following:

- The study focussed on women involved in self-help or small business activities, which may also not be representative of all women in the area.

- $\quad$ The generalisability of the results is limited as a result of the contextual nature of the design and the convenient sampling method. For example, the women in the activity groups may not represent all the women of Thulamela.

- $\quad$ The high level of education suggests that the groups may not be representative, yet the fact that the overall level of knowledge was so low may suggest that the educational level is not an indicator of knowledge regarding breast cancer.

- The data gathered was self-reported, and therefore subject to recall bias.

\section{Conclusion}

The study provided evidence that women living in Thulamela municipality in the Limpopo Province were not in the position to perform breast selfcare and were therefore unable to take responsibility for their own breast health. Their lack of knowledge of the signs and symptoms of breast cancer and breast self-examination would not enable them to prevent presenting with advanced breast cancer and maintain life, health, and wellbeing. Nurses, especially oncology nurses, have a vital role to play in promoting breast health and empowering women to apply selfcare to prevent late presentation with breast cancer. A breast health care strategy for women living in Thulamela municipality should be designed, implemented and evaluated to prevent presentation with advanced breast cancer.

\section{References}

BARRACLOUGH, J 1996: Cancer 
and emotions: a practical guide to psycho-oncology. 2nd ed. New York: John Wiley \& Sons.

BERBIGLIA, VA 2006: Orem's selfcare deficit nursing theory in practice. In: Alligood, MR \& Tomey, AM (eds). Nursing Theory. 3rd ed. St. Louis: Mosby:255-282.

BRINK, HI 2003: Fundamentals of research methodology for health care professionals. Lansdowne: Juta.

BUDAKOGLU, II; MARAL, I; OZDEMIR, A \& BUMIN, MA 2007: The effectiveness of training for breast cancer and breast self-examination in women aged 40 and over. Journal of Cancer Education. 22(2): 108-111.

BURNS, N \& GROVE, SK 2001: The practice of nursing research: conduct, critique and utilization. 4th ed. Philadelphia: Saunders.

\section{CANCERASOCIATION OF SOUTH}

AFRICA 2004: Breast cancer. Available from: http//www.cansa.org.za [Accessed on 10/01/2007].

CAPE TOWN DECLARATION ON CANCER CONTROL IN AFRICA 2006: Available from: http:// www.iaca.org/NewsCcnter/News/PDF/ ctdeclaration 1206.pdf. [Accessed: 20/ 02/2007]

CENSUS 2001: [Online]. Available from: http://www.statsa.gov.za/ census 2001 .digiAtlas/stats/ stats_langdom_NP343.html [Accessed: 13/06/2006].

CRANE-OKADO, $R$ 2001: Breast cancers. In: Otto, S. (ed.). Oncology nursing. 4th ed. St. Louis: Mosby: 113167.

DONEGAN, WL\& SPRATT, JS 1995: Cancer of the breast. St Louis: Elsevier.

DUNAR, PE; ÖZMEN, D; ÖZTÜRK, B; HASPOLAT, G; AKYILDIS, F; COBAN, S \& ÇAKIROGLU, G 2006: The knowledge and attitudes of breast self-examination and mammography in a group of women in a rural area in western Turkey. BMC Cancer, 6(43).

HAMOLSKY, D \& FACIONE, N 1999: Infiltrating breast cancer. In:
Miaskowski, C. \& Buchsel, P. (eds). Oncology nursing: assessment and clinical care. St Louis: Mosby:425-467.

IRWIN,A; VALENTINE, N; BROWN, C; LOEWENSON, R; SOLAR, BROWN, H; KOLLER, T \& VEGA, J 2006: The commission on social determinants of health: talking the social roots of health inequities. PLOS medicine, 3(6). [Online]. Available from: http://medicine.plosiournals.org/ perlserv/? request $=\mathrm{get}$ document\&doi=10\%2E $1371 \%$ [Accessed: 10/07/2006].

LEDDY, SK \& PEPPER, JM 1998: Conceptual bases of professional nursing. Philadelphia: Lippincott Williams $\&$ Wilkins

LoBIONDO-WOOD, G \& HABER, J 2006: Nursing research: methods and critical appraisal for evidence-based practice. St. Louis: Mosby.

MEDICAL RESEARCH COUNCIL 2006: FAQ: Cancer in South Africa. [Online]. Available from: http:// www.mrc.ac.za/bod/faqcancer.htm [Accessed 2007/01/10].

NAHCIVAN, NO \& SECGINLI, $S$ 2007: Health beliefs related to breast self-examination in a sample of Turkish women. Oncology Nursing Forum. $34(2): 425-432$.

OLUWATOSIN, OA 2006: Rural women's perception of breast cancer and its early-detection measures in Ibadan, Nigeria. Cancer Nursing. 29(6):461-166.

POLIT, FD \& HUNGLER, B 1997: Essentials of nursing research. 4th ed. Philadelphia: Lippincott.

PUCKREE，T; MKHIZE, M; MGOBHOZI, Z \& LIN, J 2002: African traditional healers: what health care professionals need to know. International Journal of Rehabilitation Research, 25(4):247-251.

REDDY, P 2004: Chronic diseases. [Online]. Available from: http:// www.hst.org.za/generic/29 [Accessed: 16/02/2006].

SMYKE, P 1993: Women and Health. World Development Series. New Jer- sey: Zed Books.

VOROBIOF, DA; SITAS, F \& VOROBIOF, G 2001: Breast cancer incidence in South Africa. In: Journal of clinical oncology. 19(18S), Sept.:125S-127S.

WALKER, ARP; ADAM, FI \& WAKER, BF 2004: Breast cancer in black African women: a changing situation. The Journal of the Roval Societv for the Promotion of Health. 124(2):81-85

WORLD HEALTH ORGANIZATION AND INTERNATIONAL UNION AGAINST CANCER 2005: Global action against cancer. Switzerland: WHO and IUA. 